\title{
GENERALIZED BOUNDARY VALUE PROBLEM AND ITS APPLICATION TO THE DENSITY WAVE THEORY OF SPIRAL GALAXIES
}

\author{
By Toshiniko Nishimoto
}

\section{Introduction.}

In the course of the studies on uniform asymptotic properties of the W-K-B method, the author introduced the notion of the singular curves for the equation

$$
\frac{d^{2} y}{d x^{2}}+\lambda^{2} p^{2}(x, \omega) y=0,
$$

where $p^{2}(x, \omega)$ is a polynomial of $x, \omega$ is the complex eigenvalue parameter and $\lambda$ is a positive large parameter, (see Nishimoto [10]). For any two zeros $x_{1}(\omega)$ and $x_{2}(\omega)$ of $p^{2}(x, \omega)$, the equation

$$
\operatorname{Re} \int_{x_{1}(\omega)}^{x_{2}(\omega)} p(x, \omega) d x=0
$$

determines singular curves in the complex $\omega$-plane. Here and in the following $\operatorname{Re} f$ denotes the real part of $f$ and also $\operatorname{Im} f$ the imaginary part of $f$. In this circumstance, there was a conjecture such that for each singular curve, there exist the so-called generalized boundary value problems whose eigenvalues are on or asymptotic to the singular curve. In this paper we verify this conjecture for a simple equation which plays a fundamental role in understanding the wave mechanism of the density waves of spiral galaxies. For more general equations, the analogous considerations can give us distribution of the series of discrete eigenvalues and corresponding generalized boundary value problems.

Now we explain the relation of our analysis to the density wave theory of spiral galaxies. The spiral structure is observed in many disk shaped galaxies, and theoretical explanation of this structure has been an active field of scientific research. Through the normal mode analysis based on the hydrodynamical approach, Lin and Lau [5] reduced the theory of such structure for normal spiral galaxies to a certain boundary value problem of second order ordinary differential equation. And by using the W-K-B method for this equation, they gave a consistent explanation of the wave mechanism of spiral patterns. Also the author proved the existence of the complex discrete eigenvalues of the boundary value 
problems [8]. One of the remarkable facts is that there exist several eigenvalues with small imaginary parts exhibiting a mild instability. On the other hand, Aoki, Noguchi and Iye [1] reduced the problem to an eigenvalue problem of infinite order matrix by using expansion of variables in terms of associated Legendre functions, and numerically obtained highly unstable global eigenmodes of perturbations. Moreover, many numerical calculations based on the steller dynamics or large $N$-body experiments show that highly unstable growth of perturbations can occur and give the impression of great instability, Kato et al. [7] and Matsuda and Isaka [6].

Lau [4] recently showed that the unstable and transient waves can be produced by the differential rotation of density waves.

The purpose of this paper is to predict the existence of highly unstable normal modes, by applying the generalized boundary value problems to the simple second order ordinary differential equation for which the existence of moderately unstable eigenvalues was previously proved [8]:

$$
\frac{d^{2} y}{d x^{2}}+\lambda^{2} k^{2}(x, \lambda, \omega) y=0,
$$

where

$$
k^{2}(x, \lambda, \omega)=x\left\{(\omega-c+x)^{2}+a^{2}\right\}+\frac{1}{\lambda} b k_{1}(x, \omega) .
$$

In this paper, we mostly consider a simpler equation:

$$
\frac{d^{2} y}{d x^{2}}+\lambda^{2} x(x-\omega)^{2} y=0, \quad(c-\omega \rightarrow \omega) .
$$

According to [8], the boundary conditions that

(1) $y(x)$ exponentially decays for $x<0$, and

(2) $y(x) e^{\imath \omega t}$ represents an outgoing wave as $x \rightarrow \infty$,

determine asymptotically the so-called dispersion relation or quantum condition

$$
\exp \left\{2 i \lambda \int_{0}^{\omega} \sqrt{x(x-\omega)^{2}} d x\right\}=-\frac{1}{\sqrt{ } 2} .
$$

On the other hand, it is easy to see that the singular curves for the equation (1.2) consist of half lines: $\arg \omega=2 k \pi / 5 \quad(k=0,1,2,3,4)$. Therefore we can expect the existence of a several series of eigenvalues for the equation (1.2) with appropriate boundary conditions, and some of them represent highly unstable eigenvalues.

In section 2, we give a brief account of the W-K-B method and state the generalized boundary value problems. We prove that if $\omega$ is not in a sufficiently small neighborhood of singular curves, there exists no boundary value problems for which we have discrete eigenvalues. On the other hand, to each singular 
curve we can assign boundary conditions so that there exist two series of discrete eigenvalues that are asymptotic to the singular curve. In section 3 , we consider the properties of unstable eigenfunctions on the real axis and their relations to the density wave theory of spiral galaxy.

Acknowledgement. The author wishes to thank Professor Y. Hirasawa, Dr. H. Yamada and Dr. H. Ito for many helpful discussions and valuable comments.

\section{Generalized boundary value problems.}

Following to the W-K-B theory, let us define the fundamental systems of solutions $u$ and $v$ that are characterized by a canonical domain $D, a$ turning point $a$ at the boundary of $D$ and a Stokes curve $L$ starting from $a$ and entering into the interior of $D$. They have asymptotic expansions as $\lambda \rightarrow \infty$ in the form

where

$$
\begin{aligned}
& u(x, \lambda, \omega) \cong c k(x, \omega)^{-1 / 2} \exp \{\lambda \xi(a, x, L)\}, \\
& v(x, \lambda, \omega) \cong c k(x, \omega)^{-1 / 2} \exp \{-\lambda \xi(a, x, L)\},
\end{aligned}
$$

$$
\begin{aligned}
& c=e^{-\imath \alpha}, \quad \alpha=\lim _{\substack{x \rightarrow a \\
x \in L}} \arg (\imath k)^{-1 / 2}, \\
& k(x, \omega)=\left\{x(x-\omega)^{2}\right\}^{1 / 2}, \\
& \xi(a, x, L)=i \int_{a}^{x} k(x, \omega) d x, \operatorname{Im} \xi(a, x, L)>0 \text { on } L .
\end{aligned}
$$

The branch of $\xi(a, x, L)$ is chosen so that $\operatorname{Re} \xi(a, x, L)>0$ if $x$ is on the right of $L$, oriented from $a$ to $x$. We denote thus defined fundamental system of solutions by $Y\{a, L, D\}$, or in vector form by

$$
\left(\begin{array}{l}
u \\
v
\end{array}\right)_{\{a, L, D\}}
$$

Each of the solutions $u$ and $v$ is characterized by the condition that as $x$ tends to infinity along rays in some Stokes regions of half plane type $S \subset D$, it exponentially decreases to zero, (see [2] for more details).

It is clear that the solutions satisfying the condition such as (2) of (1.3) (radiation condition) can be characterized by the subdominant nature on the appropriate Stokes region. We consider, as considered by Fedoryuk [3], the boundary value problem to find solutions $y(x, \lambda, \omega)$ of $(1.2)$ with eigenvalue parameter $\omega$ such that $\lim _{x \rightarrow \infty} y(x, \lambda, \omega)=0$ along two rays that extend to infinity in different Stokes regions of half plane type. Let us call this kind of problems as the generalized boundary value problems. Now our problem is to find two Stokes regions for which the above boundary value problem has discrete sets of eigenvalues. 
Since substitution of $x$ and $\omega$ by $x \exp 2 k \pi \imath / 5$ and $\omega \exp 2 k \pi \imath / 5(k=0,1,2$, $3,4)$ remains the equation (1.2) unchanged, it is sufficient to consider the cases where $|\arg \omega|<2 \pi / 5$. In Fig. 1, we show variations of Stokes curve configuration for $\omega$.

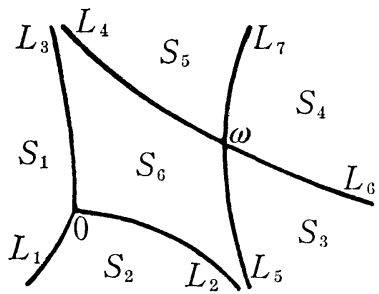

(a) $\arg \omega>0$

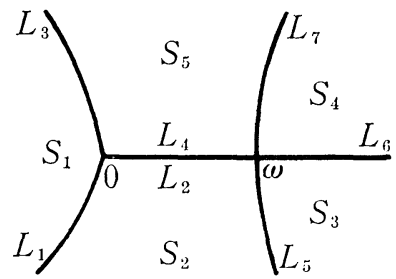

(b) $\arg \omega=0$

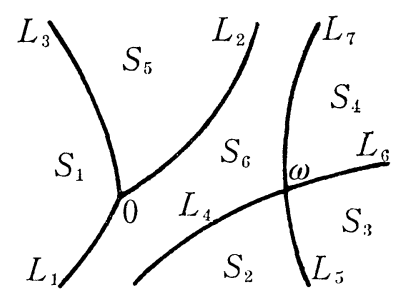

(c) $\arg \omega<0$

Fig. 1.

In the above figure, $S_{\imath}(\imath=1,2,3,4,5)$ are the Stokes regions of half plane type, and $S_{6}$ is of strip type. Let $y(x, \lambda, \omega)$ be the solution of (1.2) which has the asymptotic expansion of the form

$$
\begin{aligned}
& y(x, \lambda, \omega) \cong c k(x, \omega)^{-1 / 2} \exp \{-\lambda \xi(a, x, L)\}, \\
& \xi(a, x, L)=i \int_{a}^{x} k(x, \omega) d x .
\end{aligned}
$$

Here the branch of $k(x, \omega)$ is chosen so that $\operatorname{Im} \xi(a, x, L)$ is positive on $L$. The solution $y(x, \lambda, \omega)$ is subdominant in $S$. Now we find the maximal region $D(S)$ in which the asymptotic expansion (2.2) is valid. The region $D(S)$ is the set of points from which we can describe a rectilinear smooth curve in the following way :

(i) it is contained in $D(S)$ and extends to infinity in $S$,

(ii) $\operatorname{Re} \xi(a, x, L)$ is nondecreasing along it.

Then the maximal region $D(S)$ is obtained by making a union of a finite number of canonical domains, each of which is mapped under the mapping $x \rightarrow \xi$ $=\xi(a, x, L)$ on the whole $\xi$-plane with cuts in such a way that the image of $S$ becomes a right half plane. Thus $D(S)$ is obtained by deleting from $x$-plane some Stokes curves and Stokes regions that are determined uniquely from $S$. In the following table, we list pairs of the Stokes region and the compliment of $D(S)$. 
Table 1.

\begin{tabular}{|l|l|l|l|}
\hline$S$ & \multicolumn{3}{|c|}{ Compliment of $D(S)$} \\
\cline { 2 - 4 } & \multicolumn{1}{|c|}{$0<\arg \omega<2 \pi / 5$} & $\arg \omega=0$ & \multicolumn{1}{|c|}{$-2 \pi / 5<\arg \omega<0$} \\
\hline$S_{1}$ & $S_{4} \cup L_{2}$ & $S_{3} \cup S_{4} \cup L_{6}$ & $S_{3} \cup L_{2}$ \\
$S_{2}$ & $S_{4} \cup L_{3}$ & $S_{4} \cup L_{3}$ & $S_{4} \cup L_{3}$ \\
$S_{3}$ & $S_{5} \cup L_{1}$ & $S_{1} \cup S_{5} \cup L_{3}$ & $S_{1} \cup S_{5} \cup S_{6} \cup L_{1} \cup L_{2} \cup L_{3}$ \\
$S_{4}$ & $S_{1} \cup S_{2} \cup S_{6} \cup L_{1} \cup L_{2} \cup L_{3}$ & $S_{1} \cup S_{2} \cup L_{1}$ & $S_{2} \cup L_{3}$ \\
$S_{5}$ & $S_{3} \cup L_{1}$ & $S_{3} \cup L_{1}$ & $S_{3} \cup L_{1}$ \\
\hline
\end{tabular}

The asymptotic formula (2.2) for $\lambda \rightarrow \infty$ is uniformly valid in every compact subset of $D(S)$, and this solution decreases asymptotically zero as $x$ tends to infinity in $S$ and exponentially increases in all other Stokes regions of $D(S)$. Hence for a given Stokes region $S$, eigenvalues of our generalized boundary value problem can only exist when we choose a Stokes region not contained in $D(S)$ on which the boundary condition is imposed. In these situations, we can prove the following proposition.

PROPOSITION 1. If $\omega$ is not in a sufficiently small neighborhood of singular curves, there exists no quantum condition for any generalized boundary value problems, and so no ergenvalues.

Proof. The singular curves of the equation (1.2) are given by (1.5). We prove the above statement when $\omega$ satisfies $0<\arg \omega<2 \pi / 5$, and other cases can be proved analogously. Let $T$ and $\widetilde{T}$ be two Stokes regions of half plane type on which the boundary condition $y(x, \lambda, \omega) \rightarrow 0$ as $x$ tends to infinity in $T$ and $\tilde{T}$ is to be imposed. Following to the Table 1 we take, for example, $S_{1}$ as $T$ and $S_{4}$ as $\tilde{T}$. Let $D_{\imath}(\imath=1,2,3)$ be canonical domains defined by

$$
D_{1}=S_{1} \cup L_{1} \cup S_{2}, \quad D_{2}=S_{2} \cup L_{2} \cup S_{6} \cup L_{5} \cup S_{3}, \quad D_{3}=S_{3} \cup L_{6} \cup S_{4} .
$$

Then by using connection formulas [2], we get

$$
\begin{aligned}
& Y\left\{0, L_{2}, D_{2}\right\}=e^{\pi \imath / 6}\left(\begin{array}{cc}
-i+O\left(\lambda^{-1}\right), & 1+O\left(\lambda^{-1}\right) \\
1 & 0
\end{array}\right) Y\left\{0, L_{1}, D_{1}\right\}, \\
& Y\left\{\omega, L_{5}, D_{2}\right\}=e^{\imath \tau_{0}}\left(\begin{array}{cc}
e^{-i \tau_{1}} & 0 \\
0 & e^{\lambda \tau_{1}}
\end{array}\right) Y\left\{0, L_{2}, D_{2}\right\}, \\
& Y\left\{\omega, L_{6}, D_{3}\right\}=e^{\pi \imath / 4}\left(\begin{array}{cc}
-\sqrt{2 i}\left(1+O\left(\lambda^{-1}\right),\right. & 1+O\left(\lambda^{-1}\right) \\
1 & 0
\end{array}\right) Y\left\{\omega, L_{5}, D_{2}\right\},
\end{aligned}
$$

where

$$
\tau_{0}=\lim _{\substack{x \rightarrow 0 \\ x \in L_{2}}} \arg (i k)^{-1 / 2}-\lim _{\substack{x \rightarrow \omega \\ x \in L_{5}}} \arg (i k)^{-1 / 2},
$$


and

$$
\tau_{1}=\int_{0}^{\omega} \imath k(x, \omega) d x, \quad\left(\operatorname{Re} \tau_{1}<0\right) .
$$

If we denote elements of the fundamental systems of solutions $Y\left\{0, L_{1}, D_{1}\right\}$ and $Y\left\{\omega, L_{6}, D_{3}\right\}$ by

$$
\left(\begin{array}{l}
u_{1} \\
v_{1}
\end{array}\right)_{\left\{0, L_{1}, D_{1}\right\}} \text { and }\left(\begin{array}{c}
u_{2} \\
v_{2}
\end{array}\right)_{\left\{\omega, I_{6}, D_{3}\right\}}
$$

then the asymptotic expansion of connection matrix between them becomes

$$
\left(\begin{array}{c}
u_{2} \\
v_{2}
\end{array}\right)_{\left\{\omega, L_{6}, D_{3}\right\}}=M(\omega, \lambda)\left(\begin{array}{c}
u_{1} \\
v_{1}
\end{array}\right)_{\left\{0, L_{1}, D_{1}\right\}}
$$

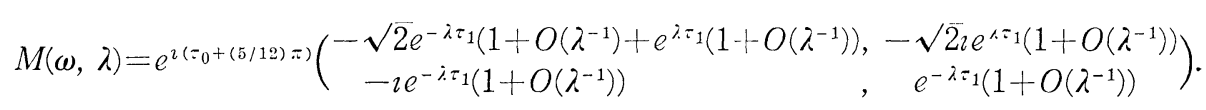

From the way of the characterization of $Y\left\{0, L_{1}, D_{1}\right\}$, the solution $v_{1}(x, \lambda, \omega)$ is subdominant in the Stokes region $S_{1} \subset D_{1}$, and the solution $u_{2}(x, \lambda, \omega)$ is also recessive in $S_{4} \subset D_{3}$. Therefore to exist a solution which is subdominant on $S_{1}$ and $S_{4}$, it is necessary and sufficient that the 1-1 element of the matrix (2.3) vanishes: that is

$$
-\sqrt{2} e^{-\lambda \tau_{1}}\left(1+O\left(\lambda^{-1}\right)+e^{\lambda r_{1}}\left(1+O\left(\lambda^{-1}\right)\right)=0 .\right.
$$

But this is impossible for sufficiently large $\lambda$ because of $\operatorname{Re} \tau_{1}<0$. Thus the statements of the Proposition 1 is proved for $S_{1}$ and $S_{4}$, and other pairs of $T$ and $\widetilde{T}$ from the Table 1 lead to the same conclusion.

From the above proposition, eigenvalues can only exist when $\omega$ is in a sufficiently small neighborhood of a singular curve: $\arg \omega=0$. In this case, there are several couples of $S_{\imath}, S_{\jmath}$ as $T$, and $\widetilde{T}$, but we can prove that only two of them give us the so called quantum condition.

PROPOSITION 2. There exist two series of discrete engenvalues that are asymptotic to the singular curve: $\arg \omega=0$.

Proof. Using the notations in Fig. 1(b), we define canonical domains $D_{\imath}$ $(i=1,2,3,4)$ :

$$
\begin{array}{ll}
D_{1}=\left\{S_{1} \cup L_{1} \cup S_{2}\right\}, & D_{2}=\left\{S_{2} \cup L_{2} \cup S_{5}\right\}, \\
D_{3}=\left\{S_{2} \cup L_{5} \cup S_{3}\right\}, & D_{4}=\left\{S_{3} \cup L_{6} \cup S_{4}\right\} .
\end{array}
$$

As before, we calculate leading terms of connection matrices between two fundamental systems of solutions defined on adjacent canonical domains: 


$$
\begin{aligned}
& Y\left\{0, L_{2}, D_{2}\right\}=e^{\pi \imath / 6}\left(\begin{array}{cc}
-i\left(1+O\left(\lambda^{-1}\right)\right), & 1+O\left(\lambda^{-1}\right) \\
0 & 0
\end{array}\right) Y\left\{0, L_{1}, D_{1}\right\}, \\
& Y\left\{\omega, L_{2}, D_{2}\right\}=e^{\imath \tau_{0}}\left(\begin{array}{cc}
0, & e^{\lambda \tau_{1}} \\
e^{-\lambda \tau_{1}}, & 0
\end{array}\right) Y\left\{0, L_{2}, D_{2}\right\}, \\
& Y\left\{\omega, L_{3}, D_{3}\right\}=e^{\pi \imath / 4}\left(\begin{array}{cc}
-\sqrt{2 i}\left(1+O\left(\lambda^{-1}\right)\right), & 1+O\left(\lambda^{-1}\right) \\
1 & 0
\end{array}\right) Y\left\{\omega, L_{2}, D_{2}\right\}, \\
& Y\left\{\omega, L_{6}, D_{4}\right\}=e^{\pi \imath / 4}\left(\begin{array}{cc}
-\sqrt{2 i}\left(1+O\left(\lambda^{-1}\right)\right), & 1+O\left(\lambda^{-1}\right) \\
1 & 0
\end{array}\right) Y\left\{\omega, L_{3}, D_{3}\right\},
\end{aligned}
$$

where

$$
\begin{aligned}
& \tau_{0}=\lim _{\substack{x \rightarrow 0 \\
x \in L_{2}}} \arg (i k)^{-1 / 2}-\lim _{\substack{x \rightarrow \omega \\
x \in L_{2}}} \arg (i k)^{-1 / 2} \\
& \tau_{1}=\xi\left(0, \omega, L_{2}\right), \quad \operatorname{Im} \tau_{1}>0 \text { on } L_{2} .
\end{aligned}
$$

Let us put

$$
Y\left\{0, L_{1}, D_{1}\right\}=\left(\begin{array}{c}
u_{1} \\
v_{1}
\end{array}\right)_{\left\{0, L_{1}, D_{1}\right\}}, \quad Y\left\{\omega, L_{6}, D_{4}\right\}=\left(\begin{array}{c}
u_{2} \\
v_{2}
\end{array}\right)_{\left\{\omega, L_{6}, D_{4}\right\}},
$$

then we have

$$
\left(\begin{array}{l}
u_{2} \\
v_{2}
\end{array}\right)_{\left\{\omega, L_{6}, D_{4}\right\}}=M(\omega, \lambda)\left(\begin{array}{l}
u_{1} \\
v_{1}
\end{array}\right)_{\left\{0, L_{1}, D_{1}\right\}},
$$

with

$$
M(\omega, \lambda)=e^{\imath\left(\tau_{0}+(2 / 3) \pi\right)}\left(\begin{array}{lc}
-\sqrt{2} e^{-\lambda \tau_{1}}\left(1+O\left(\lambda^{-1}\right)\right)+e^{\lambda \tau_{1}}\left(-1+O\left(\lambda^{-1}\right)\right), & -\sqrt{2} 2 e^{-\lambda \tau_{1}}\left(1+O\left(\lambda^{-1}\right)\right) \\
-i e^{-\lambda \tau_{1}}\left(1+O\left(\lambda^{-1}\right)\right)-\sqrt{2} i e^{\lambda \tau_{1}}\left(1+O\left(\lambda^{-1}\right)\right), & e^{-\lambda \tau_{1}}\left(1+O\left(\lambda^{-1}\right)\right)
\end{array}\right) .
$$

The solution $v_{1}$ is subdominant in the Stokes region $S_{1}$, and the solutions $u_{2}$ and $v_{2}$ are also recessive in $S_{4}$ and $S_{3}$ respectively. Then to exist solutions of the generalized boundary value problems with $T=S_{1}$ and $\tilde{T}=S_{3}$, it is necessary and sufficient that

$$
-\imath e^{-\lambda \tau_{1}}\left(1+O\left(\lambda^{-1}\right)\right)-\sqrt{2} i e^{\lambda \tau_{1}}\left(1+O\left(\lambda^{-1}\right)\right)=0,
$$

and also with $T=S_{1}$ and $\widetilde{T}=S_{4}$

$$
-\sqrt{2} e^{-\lambda \tau_{1}}\left(1+O\left(\lambda^{-1}\right)\right)+e^{\lambda \tau_{1}}\left(1+O\left(\lambda^{-1}\right)\right)=0 .
$$

Since $\operatorname{Re} \tau_{1}$ is zero or almost zero, $\exp \left(-\lambda \tau_{1}\right)$ and $\exp \left(\lambda \tau_{1}\right)$ are comparable in magnitude and then the equations (2.6) and (2.7) can give us quantum conditions of generalized boundary value problems. As the first approximation of quantum conditions, we get

$$
2 i \lambda \int_{0}^{\omega}\left\{x(x-\omega)^{2}\right\}^{1 / 2} d x=(2 n+1) \pi \imath-\log \sqrt{2} \quad(n=0,1,2, \cdots),
$$

and 


$$
2 i \lambda \int_{0}^{\omega}\left\{x(x-\omega)^{2}\right\}^{1 / 2} d x=(2 n) \pi \imath+\log \sqrt{2} \quad(n=0,1,2, \cdots) .
$$

Here we choose the branch $\left\{x(x-\omega)^{2}\right\}^{1 / 2}$ so that

$$
\operatorname{Re} \int_{0}^{\omega}\left\{x(x-\omega)^{2}\right\}^{1 / 2} d x>0
$$

and then

$$
2 i \lambda \int_{0}^{\omega}\left\{x(x-\omega)^{2}\right\}^{1 / 2} d x=\frac{8}{15} i \lambda \omega^{5 / 2}
$$

These give us two series of discrete and almost real eigenvalues, and it is easy to see that other choice of two Stokes regions as $T$ and $\tilde{T}$ from the Table 1 can not define quantum conditions.

Now let us consider the other singular curves. To do so, we make substitutions :

$$
x=\hat{x} \exp \frac{2}{5} k \pi \imath, \quad \omega=\hat{\omega} \exp \frac{2}{5} k \pi \imath, \quad(k=0,1,2,3,4) .
$$

Then the differential equation (1.2) becomes

$$
\frac{d^{2} y}{d \hat{x}^{2}}+\lambda^{2} \hat{x}(\hat{x}-\hat{\omega})^{2} y=0 .
$$

Applying the results obtained in the preceding paragraphs to the equation (2.11), we get another series of discrete eigenvalues with respect to the original equation (1.2).

Let the boundary conditions be as follow:

$$
\begin{array}{ll}
\lim _{\substack{x \rightarrow \infty \\
x \in T_{k}, \widetilde{T}_{k}}} y(x)=0 & T_{k}: \frac{4}{5} \pi+\frac{2}{5} k \pi<\arg x<\frac{6}{5} \pi+\frac{2}{5} k \pi, \\
& \widetilde{T}_{k}:-\frac{2}{5} \pi+\frac{2}{5} k \pi<\arg x<\frac{2}{5} k \pi,
\end{array}
$$

and

$$
\begin{aligned}
& \lim _{k}^{\prime}: \frac{2}{5} k \pi<\arg x<\frac{2(k+1)}{5} \pi, \\
& \lim _{\substack{x \rightarrow \infty \\
x \in T^{\prime}, \widetilde{T}_{k}^{\prime}}} y(x)=0 \quad \widetilde{T}_{k}^{\prime}: \frac{4}{5} \pi+\frac{2}{5} k \pi<\arg x<\frac{6}{5} \pi+\frac{2}{5} k \pi,
\end{aligned}
$$

Corresponding to (2.8), the asymptotic expression of the quantum condition for the boundary value problem (2.11) and (2.12) is

$$
2 i \lambda \int_{0}^{\hat{\omega}}\left\{x(x-\hat{\omega})^{2}\right\}^{1 / 2} d x=(2 n+1) \pi \imath-\log \sqrt{2}, \quad(n=0,1,2, \cdots),
$$


and for (2.11) and (2.13), corresponding to (2.9) we have

$$
2 i \lambda \int_{0}^{\hat{\omega}}\left\{x(x-\hat{\omega})^{2}\right\}^{1 / 2} d x=(2 n) \pi \imath+\log \sqrt{2}, \quad(n=0,1,2, \cdots),
$$

where

$$
\hat{\omega}=\omega_{k} \exp \left(-\frac{2}{5} k \pi i\right), \quad(k=0,1,2,3,4) .
$$

Therefore we obtained for each singular curve two series of discrete eigenvalues $\omega_{k}^{(n)}$ and $\omega_{k}^{\prime(n)}$ that are asymptotic to the singular curve as $n \rightarrow \infty$.

\section{Highly unstable normal modes of density waves.}

Now we apply those results obtained in section 2 to the physical problems of the density wave theory. One of the boundary conditions (1.3) (2): the outgoing wave condition at $x=\infty$ can be rewritten as the subdominant condition at the Stokes region $S_{3}$. The quantum condition (2.8) is a part of the results obtained in [8]. On the other hand, the quantum condition (2.9) determined by the subdominant condition on the Stokes region $S_{4}$ can be obtained by the ingoing wave condition at $x=\infty$. The eigenvalues determined by (2.9) have small negative imaginary parts and then may represent stable normal modes. Highly unstable normal modes can be obtained from the quantum condition (2.14) and (2.15) with $k=1$.

Let $\omega_{1}^{(n)}$ and $y\left(x, \lambda, \omega_{1}^{(n)}\right)$ be the eigenvalues and corresponding eigen-functions that are determined by the subdominant condition at $S_{1}$ and $S_{3}$ (see Fig. 2), and also $\omega_{1}^{\prime(n)}$ and $y\left(x, \lambda, \omega_{1}^{\prime(n)}\right)$ determined by $S_{1}$ and $S_{4}$.

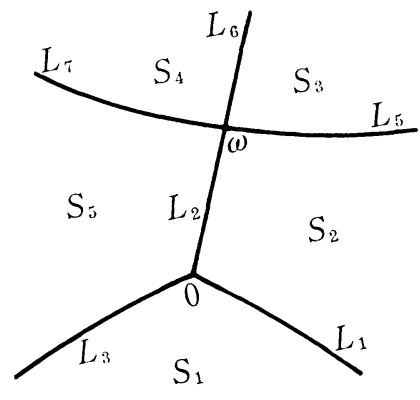

Fig. 2

These eigenvalues $\omega_{1}^{(n)}$ and $\omega_{1}^{(n)}$ are close to the singular curve arg $\omega=2 \pi / 5$ and then exhibit highly unstable normal modes. These eigenfunctions $y\left(x, \lambda, \omega_{1}^{(n)}\right)$ and $y\left(x, \lambda, \omega_{1}^{\prime(n)}\right)$ are expressed as

$$
\begin{aligned}
& y\left(x, \lambda, \omega_{1}^{(n)}\right)=v_{1}\left(x, \lambda, \omega_{1}^{(n)}\right) \cong c_{1} k\left(x, \omega_{1}^{(n)}\right)^{-1 / 2} \exp \left\{-\lambda \xi\left(0, x, L_{1}\right)\right\}, \\
& y\left(x, \lambda, \omega_{1}^{\prime(n)}\right)=v_{1}\left(x, \lambda, \omega_{1}^{\prime(n)}\right) \cong c_{2} k\left(x, \omega_{1}^{\prime(n)}\right)^{-1 / 2} \exp \left\{-\lambda \xi\left(0, x, L_{1}\right)\right\},
\end{aligned}
$$


where

$$
\xi\left(0, x, L_{1}\right)=i \int_{0}^{x} k(x, \omega) d x, \quad \operatorname{Im} \xi>0 \text { on } L_{1}
$$

in the canonical domain $S_{1} \cup L_{1} \cup S_{2}$. Here and hereafter $c_{2}$ denotes some constants with $\left|c_{\imath}\right|=1$. These solutions behave as dominant functions on the negative real axis. To get their asymptotic expansions in the neighborhood of the positive real axis, we first express them by the fundamental systems of solutions $Y\left\{\omega, L_{6}, D_{4}\right\}=\left(\begin{array}{c}u_{2} \\ v_{2}\end{array}\right)$ defined on the canonical domain $D_{4}=S_{3} \cup L_{6} \cup S_{4}$. From the connection formula (2.5), and the quantum conditions (2.6) and (2.7), we have

$$
\begin{aligned}
& y\left(x, \lambda, \omega_{1}^{(n)}\right)=c_{3} e^{\lambda \tau_{1}}\left(1+O\left(\lambda^{-1}\right)\right) v_{2}\left(x, \lambda, \omega_{1}^{(n)}\right), \quad \tau_{1}=\xi\left(0, \omega_{1}^{(n)}, L_{2}\right), \\
& y\left(x, \lambda, \omega_{1}^{\prime(n)}\right)=c_{4} \frac{1}{\sqrt{ } 2} e^{\lambda \tau_{1}^{\prime}}\left(1+O\left(\lambda^{-1}\right)\right) u_{2}\left(x, \lambda, \omega_{1}^{\prime(n)}\right), \quad \tau_{1}^{\prime}=\xi\left(0, \omega_{1}^{\prime(n)}, L_{2}\right) .
\end{aligned}
$$

Let $\left(\begin{array}{l}u_{3} \\ v_{3}\end{array}\right)$ be the fundamental system of solutions defined on $D_{3}=S_{2} \cup L_{5} \cup S_{3}$ :

then we have

$$
\begin{aligned}
& u_{3} \cong c_{5} k(x, \omega)^{-1 / 2} \exp \left\{\lambda \xi\left(\omega, x, L_{5}\right)\right\}, \\
& v_{3} \cong c_{5} k(x, \omega)^{-1 / 2} \exp \left\{-\lambda \xi\left(\omega, x, L_{5}\right)\right\},
\end{aligned}
$$

$$
\left(\begin{array}{l}
u_{2} \\
v_{2}
\end{array}\right)=e^{\pi 2 / 4}\left(\begin{array}{cc}
-\sqrt{2 i}\left(1+O\left(\lambda^{-1}\right)\right), & 1+O\left(\lambda^{-1}\right) \\
1 & 0
\end{array}\right)\left(\begin{array}{l}
u_{3} \\
v_{3}
\end{array}\right)
$$

Thus the eigenfunctions can be expressed on the neighborhood of the positive real axis as follows:

$$
y\left(x, \lambda, \omega_{1}^{(n)}\right)=c_{6} e^{\lambda \tau_{1}} v_{3} \cong c_{6} e^{\lambda \tau_{1}} k\left(x, \omega_{1}^{(n)}\right)^{-1 / 2} \exp \left\{-\lambda \xi\left(\omega_{1}^{(n)}, x, L_{5}\right)\right\},
$$

and

$$
\begin{aligned}
& y\left(x, \lambda, \omega_{1}^{\prime(n)}\right)=\frac{1}{\sqrt{ } 2} e^{\lambda \tau_{1}^{\prime}}\left\{c_{7} \sqrt{2}\left(1+O\left(\lambda^{-1}\right)\right) u_{3}+c_{8}\left(1+O\left(\lambda^{-1}\right)\right) v_{3}\right\}
\end{aligned}
$$

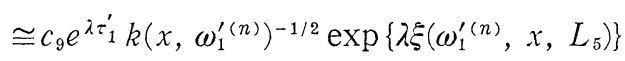

$$
\begin{aligned}
& +c_{10} \frac{1}{\sqrt{ } 2} e^{\lambda \tau_{1}^{\prime}} k\left(x, \omega_{1}^{\prime(n)}\right)^{-1 / 2} \exp \left\{-\lambda \xi\left(\omega_{1}^{\prime(n)}, x, L_{5}\right)\right\} .
\end{aligned}
$$

Since the Stokes curve $L_{5}$ approaches the positive real axis as $x \rightarrow \infty$, (3.5) and (3.6) mean that the eigenfunctions represent waves for sufficiently large $x$.

As in [8], p. 19, let us define group velocity $c_{g}$ by

$$
c_{g}=\operatorname{Re}\left\{\frac{\partial k}{\partial \omega}\right\}^{-1}=-\operatorname{Re}\left\{-\frac{k(x, \omega)}{x(x-\omega)}\right\} .
$$

The branch of $k(x, \omega)$ is so chosen that $\operatorname{Re} k(x, \omega)>0$ on $L_{5}$, and then $u_{3}$ repre- 
sents an ingoing wave and $v_{3}$ an outgoing wave on real axis for sufficiently large $x$. Thus from (3.5) and (3.6), the eigenfunction $y\left(x, \lambda, \omega_{1}^{(n)}\right)$ is an outgoing wave and $y\left(x, \lambda, \omega_{1}^{\prime(n)}\right)$ is a linear combination of an outgoing wave and an ingoing wave. Because these highly unstable eigenfunctions are dominant on the negative real axis, they can not be characterized by giving asymptotic conditions on the real axis.

The equation (1.2) that we have studied is quite simple, but because of the analyses of the previous papers [8] and [9] we can expect that more realistic equations describing the density waves have eigenvalues of the highly unstable nature as those in this paper, and this may help understanding discrepancy underlying between asymptotic method and numerical analysis.

\section{REFERENCES}

[1] Aoki, S., M. Noguchi And M. Iye, Global instability of polytropic gaseous disk galaxies with Toomres density distribution, Astro. Soc. Japan. 31 (1979), 737774 .

2] Evgrafov, M. A. And M. V. Fedoryuk, Asymptotic behavior of the solution of the equation $w^{\prime \prime}(\boldsymbol{z})-p(\boldsymbol{z}, \lambda) w(z)=0$ in the complex domain, Russian Math. Surveys, 21 (1966), 1-48.

[3] FEDORYUK, M.V., Asymptotic behavior of the discrete spectrum of the operator $-w^{\prime \prime}(x)+\lambda^{2} p(x) w(x)$, Mat. Sb. 68 (1965), 81-110.

[4] LAU, Y. Y., Waves in a sheared medium, Phys. Fluids (1981).

[5] LIN, C.C. AND Y.Y. LAU, Density wave theory of spiral structure of galaxies, Studies in Appl. Math., 60 (1979), 97-163.

[6] Matsuda, T. ANd H. Isaka, Response of gas to an ovally deformed gravitational potential of a disk galaxy, Progress of Theor. Phys. 64 (1980), 1265-1279.

7] Nishida, M., M. Yoshizawa, Y. Watanabe, S. Inagaki and S. Kato, Vlasov simulation of steller disks, 11., Nonaxisymmetric case, Publ. Astro. Soc. Japan 33 (1981), 567-582.

[8] Nishimoto, T., Global dispersion relation for density waves in a certain simplified model of disk shaped galaxy. Studies in Appl. Math., 60 (1979), 11-26.

[9] Nishimoto, T., Asymptotic reduction of a certain boundary value problem arising from the density wave theory of spiral galaxies, Koda1 Math. J. 4 (1981), 152180.

[10] Nishimoto, T., Uniform asymptotic properties of the W-K-B method, Kodai Math. J., 4 (1981), 71-81.

Department of Methematics

Tokyo Institute of Technology 